\title{
Update Opportunistic Infections of the Central Nervous System in Patients with latrogenic Immunosuppression
}

\section{(ब)(1) $\odot \ominus$}

Authors

Gabriele Arendt ${ }^{1}$, Matthias Maschke ${ }^{2}$

Affiliations

1 Department of Neurology, University Hospital Düsseldorf, Düsseldorf, Germany

2 Department of Neurology, Barmherzige Brüder Hospital, Trier, Germany

Key words

Opportunistic infections, central nervous system (CNS), iatrogenic immunosuppression and immunomodulation, typical infectious bodies

\author{
Bibliography \\ DOI https://doi.org/10.1055/s-0043-118924 \\ Neurology International Open 2017; 1: E316-E325 \\ (c) Georg Thieme Verlag KG Stuttgart · New York \\ ISSN 2511-1795 \\ Correspondence \\ Prof. Dr. med. Gabriele Arendt \\ Department of Neurology, University of Duesseldorf, \\ Medical Faculty \\ Moorenstr. 5 \\ 40225 Düsseldorf \\ Germany \\ Gabriele.Arendt@uni-duesseldorf.de
}

\begin{abstract}
Opportunistic infections of the central nervous system (CNS) with bacteria, parasites, fungi or viruses due to iatrogenic immunosuppression are of immense importance because of rising numbers of organ transplantations and immunomodulating treatments. Besides transplantation medicine, the most frequently involved medical subspecialties are dermatology (interferons, rituximab, fingolimod, among others), hematology/oncology (rituximab, among others), neurology (beta-interferon, glatiramer acetate, natalizumab, rituximab, teriflunomide, fingolimod, alemtuzumab, daclizumab, among others) and rheumatology (rituximab).

In Europe, typical infections affecting the immunocompromised host are due to herpes viruses and, especially in immunomodulated patients, JC virus (JCV); frequently occurring fungi are Aspergillus fumigatus, Candida albicans and Cryptococcus neoformans. An important parasite is Toxoplasma gondii (TG). Typical bacterial infections of the immunocompromised patient are caused by Nocardia asteroides, Listeria monocytogenes and Mycobacterium tuberculosis. Modern diagnostic and therapeutic procedures will be described.
\end{abstract}

\section{Introduction}

In the age of organ transplantation medicine and immunosuppressive/immunomodulating therapies, infections of the central nervous system have become a major concern in medicine. The estimated total incidence of infectious meningoencephalitis in Europe is 1.5 to 7 cases per 100000 population [1]. However, precise incidence data-especially with regard to immunocompromised patients-are not available [2]. Besides transplantation medicine, dermatology (interferons, rituximab, fingolimod, among others), hematology/oncology (rituximab, among others), neurology (beta interferon, glatiramer acetate, natalizumab, rituximab, teriflunomide, fingolimod, alemtuzumab, daclizumab, among others), and rheumatology (rituximab, among others) are among the specialties that contribute, by using their modern treatments, to a continuously increasing population of patients at risk for infections of the central nervous system (CNS) caused by rare pathogens. Among these pathogens are bacteria, fungi, parasites, and viruses, causing acute, subacute and chronic disease. The central nervous system is to some degree protected by the blood-brain barrier against pathogens attempting to intrude from the outside. However, the expression of major histocompatibility complex (MHC) molecules on microglial cells and perivascular macrophages is significantly weaker compared to other organs resulting in lower antigen presentation of the pathogen and consequently higher survival chances of the respective infectious agent [3].

- Table 1 lists the most common opportunistic cerebral infections in iatrogenically immunocompromised patients.

Opportunistic pathogens exploit various mechanisms to cross the blood-brain barrier; for example, rabies and herpes simplex viruses gain access to the central nervous system via peripheral nerves and retrograde axonal transport, while bacteria and fungi penetrate directly from the bloodstream into the brain by binding 
- Table 1 The most common opportunistic pathogens.

\begin{tabular}{|l|l|}
\hline Bacteria & $\begin{array}{l}\text { Nocardia asteroides } \\
\text { Listeria monocytogenes } \\
\text { Mycobacterium tuberculosis }\end{array}$ \\
\hline Parasites & Toxoplasma gondii \\
\hline Fungi & $\begin{array}{l}\text { Aspergillus fumigatus } \\
\text { Candida albicans } \\
\text { Cryptococcus neoformans }\end{array}$ \\
\hline Viruses & $\begin{array}{l}\text { Herpes viruses } \\
\text { C virus }\end{array}$ \\
\hline
\end{tabular}

via surface antigens to the blood-brain barrier's tight junctions, thereby opening these up for the intruding pathogens. Toxoplasma gondii triggers an inflammatory response of the blood-brain barrier's endothelial cells, resulting in increased permeability.

Immunocompromised patients presenting with neurological symptoms should alert clinicians to the possibility of meningoencephalitis caused by rare pathogens. These patients have a poor prognosis, particularly if increased intracranial pressure, severely altered mental status or seizures are present [4]. A patient's survival chance is increased by an early diagnosis.

In general, patients with compromised cellular immunity and space-occupying pulmonary and cerebral lesions are likely to suffer from either nocardiosis, aspergillosis, cryptococcosis or toxoplasmosis. Patients with T-cell defects and meningitis caused by a rare pathogen usually suffer from listeriosis, cryptococcosis, toxoplasmosis or cytomegalovirus infection.

Cryptococcal meningoencephalitis is diagnosed using antigen detection in cerebrospinal fluid (CSF), while bacterial cerebral infections, including tuberculosis, are diagnosed using polymerase chain reaction and/or cultures. However, even today abscess-forming space-occupying lesions in the CNS require tissue biopsies to confirm the diagnosis. In addition, the supplemental use of modern radiological imaging techniques, such as PET-CT and dynamic susceptibility contrast = DSC-MRI, may be indicated [5] ( Fig. 1).

In summary, cerebral infections of iatrogenically. immunocompromised patients are similar in terms of clinical presentation and findings of supplementary diagnostic testing to those in patients with normal immunity, but the rare pathogens discussed below are more commonly detected in immunocompromised patients [7].

\section{Bacterial infections}

The most common bacterial pathogens causing infection in immunocompromised patients are Nocardia asteroides, Listeria monocytogenes and Mycobacterium tuberculosis. ( Table 1)

\section{Nocardia asteroides}

Nocardia asteroides is a bacterium causing cerebral microabscesses, especially in solid-organ transplant recipients. As it is very frequently not detected during standard diagnostic workup, invasive testing (brain biopsy) is required; in the biopsy specimens obtained, gram-positive rods can be easily identified.

Common clinical features include preceding pneumonia and during the course of the disease fever, headache, mental alteration and focal neurological deficits. Frequently, these patients also develop skin abscesses; thus, a dermatologist should be consulted in all patients with suspected nocardiosis [8].

Findings of cerebrospinal fluid analysis are usually normal. Additional imaging studies (cMRI) show cerebral, ring-enhancing lesions representing microabscesses ( $\mathbf{F i g . ~ 2 )}$ ).

In about $85 \%$ of cases, the infection is only diagnosed post mortem. Nocardiosis is not only typical for iatrogenically immunocompromised patients, but also for intravenous drug addicts, filling their syringes with water from puddles, as for this microorganism mud puddles are an ideal culture medium.

With early diagnosis, treatment with trimethoprim-sulfamethoxazole or gyrase inhibitors [9] should be initiated; however, frequently the patient's life cannot be saved.

Other antibiotics that can be used include linezolid and amikacin.

\section{Listeria monocytogenes}

Listeria monocytogenes is a gram-positive, anaerobic bacterium, causing acute meningoencephalitis, predominantly affecting the brain stem in many cases [10].

While a rapid decline in the level of consciousness and at times severe nuchal rigidity are common findings, focal neurological deficits are rare.

CSF analysis usually reveals granulocytic pleocytosis of $>1000$ cells, often a marked increase in protein $(>2 \mathrm{~g} / \mathrm{dl}$ ) and a decrease in glucose levels with elevated lactate, in line with bacterial infection.

Imaging shows meningeal contrast medium enhancement and very often intraparenchymal lesions, mainly in the brainstem. Especially the combination of brainstem lesions and meningeal contrast medium enhancement should raise suspicion of listeria meningoencephalitis.

Diagnosis is confirmed by Gram staining, detection of listeria DNA by polymerase-chain reaction in CSF and cultures.

High-dose moxifloxacin therapy over a period of more than 3 weeks should be initiated [11] which is more effective than ampicillin, another frequently used antibiotic. However, in patients with confirmed listeria meningoencephalitis, first-line therapy is the combination of ampicillin and gentamycin.

Obstructive hydrocephalus (hydrocephalus occlusus) is a common complication caused by the marked increase in CSF protein levels, potentially requiring shunt placement. However, one should be aware that the high CSF protein concentrations often lead to shunt occlusion; thus, first it should be attempted to reduce intracerebral pressure by repetitive lumbar punctures.

\section{Mycobacterium tuberculosis}

In contrast to most other bacterial infections, Mycobacterium tuberculosis-induced meningoencephalitis is a chronic condition.

Over a period of several weeks to months, patients experience subfebrile temperatures with evening peaks and headaches only partly controlled by analgetics.

However, tuberculous meningoencephalitis may take an acute course in patients receiving anti-TNF-alpha therapy. 


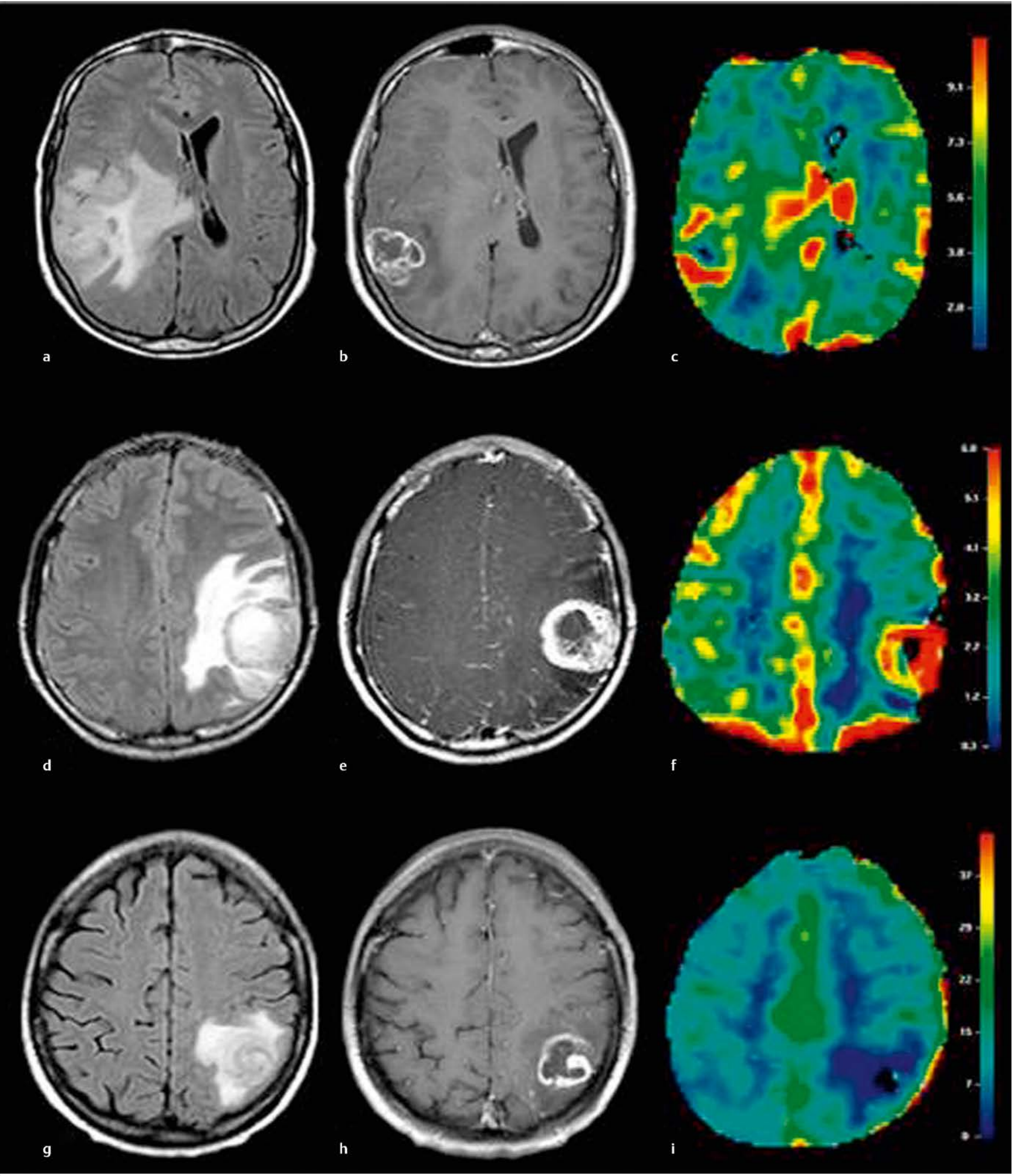

- Fig. 1 Use of modern neuroradiological techniques (DSC-MRI) to distinguish cerebral toxoplasmosis from a lymphoma; the lymphoma is metabolically active showing bright red, while toxoplasmosis is a "cold » abscess» showing blue-modified according to Dibbie et al., 2017 [6]. 
To diagnose the disease, the QuantiFERON test is used which is comparable to the tuberculin skin test (TST), according to a study with US Navy recruits [12].

- Table 2 lists Mycobacterium tuberculosis-induced neurological complications.

The most common complication of Mycobacterium tuberculosis CNS infection is purulent tuberculous meningitis. Cardinal features are headache, seizures and mental alteration; basal meningitis with paresis of cranial nerves IX-XII, described as "classical" presentation in older textbooks, is in fact very rare. About one-quarter of patients develop nuchal rigidity; in only about $12 \%$ of cases, tuberculous meningitis is preceded by extracerebral manifestations of the disease.

Especially older patients are often afebrile. Occasionally, papillary edema is present. Ophthalmoscopy reveals choroidal tuberculoma, presenting as yellow lesions with raised margins which may occur solitary or in clusters. Increasing loss of vison is suspicious of arachnoidal involvement or a tuberculoma compressing the optic nerve. Involvement of cranial nerves III, IV and VI or internuclear ophthal-

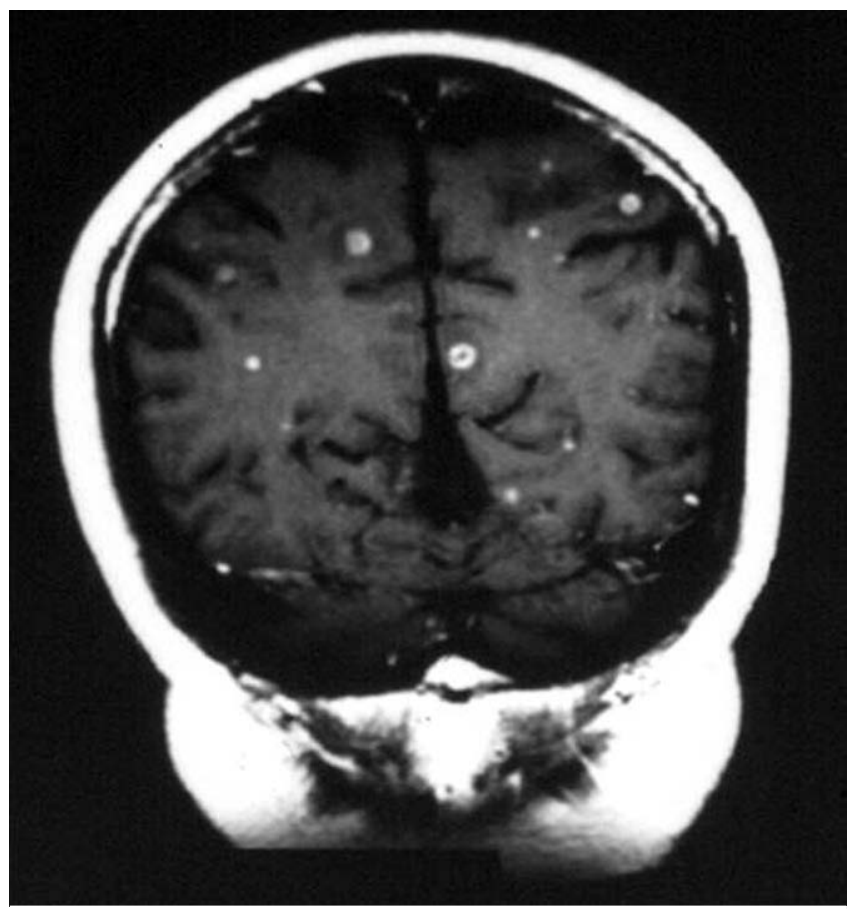

- Fig. 2 cMRI of a patients with cerebral nocardiosis; multiple ring-enhancing microabsceses. moplegia (INO) is frequent. Most commonly, cranial nerve $\mathrm{VI}$ is affected, followed by cranial nerves III, IV, VII, II, VIII, X, XI, and XII.

Choreatic, hemiballistic and athetoid movement disorders are rarely observed. The latter are frequently caused by underlying tuberculous vasculitis and tend to persist even after successful treatment of tuberculosis.

Magnetic resonance imaging (MRI) shows meningeal contrast medium enhancement along with meningoencephalitis, while cerebral ring-enhancing lesions correlate with tuberculous brain abscesses [13]

Cerebrospinal fluid analysis shows lympho-monocytic pleocytosis and significantly increased protein levels ( $>5 \mathrm{~g} / \mathrm{dl}$ ) as a laboratory correlate of chronic meningoencephalitis; the latter is responsible for the typically observed "spider's web clot". In addition, decreased CSF glucose and increased lactate levels are found. For pathogen detection, Ziehl-Neelsen staining and polymerase chain reactions are used.

Similar to listeriosis, obstructive hydrocephalus is a common complication in patients with tuberculous meningoencephalitis, requiring neurosurgical treatment.

Anti-tuberculosis treatment is determined by the antibiotic resistance profile of the responsible pathogen.

The management of infection with Mycobacterium tuberculosis susceptible to first-line anti-tuberculosis medications starts with a high-intensity 2-months treatment phase with the following substances:

isoniazid (INH) + rifampicin, ethambutol and pyrazinamide.

This is followed by a 9-13 months continuation phase with INH and rifampicin or rifabutin.

$\mathrm{MD}$ (multidrug)- and XD (extensively drug)-resistant mycobacteria are treated with a combination of $\mathrm{INH}$, rifampicin or rifabutin plus pyrazinamide and ethambutol plus moxifloxacin or levofloxacin.

Treatment has to be adapted to the results of antibiotic susceptibility testing. Here, it is important to take into consideration that multiple resistances can be expected in migrants from Eastern Europe.

In patients with diffuse meningoencephalitis, the anti-tuberculosis drugs are combined with dexamethasone to prevent hydrocephalus [14].

In addition, vitamin B complex and allopurinol should be administered to prevent INH-induced polyneuropathy and ethambutol-induced hyperuricemia, respectively. Furthermore, regular ophthalmological checks should be performed to enable early detection of ethambutol-induced optic neuropathy [15].

- Table 2 Mycobacterium tuberculosis-induced neurological complications (modified according to Arendt, 2007).

\begin{tabular}{|l|l|l|}
\hline Meningeal & \multicolumn{1}{|c|}{ Cerebral } \\
\hline Purulent tuberculous meningitis & Tuberculoma & Spinal tuberculoma \\
\hline Disseminated miliary tuberculosis & Tuberculous brain abscess & Spinal tuberculous abscess \\
\hline Inflammatory, caseating meningitis & Cerebral infarction & Necrotizing myelopathy \\
\hline Proliferative meningitis & & Tuberculous Myeloradiculitis \\
\hline Serous tuberculous meningitis & & \\
\hline
\end{tabular}


In immunocompromised patients, Mycobacterium tuberculosis infection is often associated with immune reconstitution inflammatory syndrome (IRIS). After treatment for tuberculosis, activation of inflammatory cells results in deterioration of the patient's clinical condition, a potentially life-threatening complication. Steroids may be added to the regimen while anti-tuberculosis treatment is continued; however, whether this approach is successful remains to be seen.

In summary, cerebral complications of tuberculosis are life-threatening and require immediate and consistent therapeutic intervention [16].

\section{Protozoa}

\section{Cerebral toxoplasmosis}

Cerebral toxoplasmosis is typically seen in patients with HIV infection, but can also occur in iatrogenically immunocompromised patients, especially in kidney transplant recipients. It is caused by Toxoplasma gondii and results from reactivation of cysts which had until then remained dormant in brain tissue (tachyzoites).

Seroprevalence of antibodies to Toxoplasma gondii (T. g.) varies widely between geographical regions; while seroprevalence in the US population is $11 \%$, it is $50-80 \%$ in the European population, reflecting dietary habits (the pathogen is found in raw pork, which is significantly less consumed in the US compared to Europe) and various habits related to animal keeping (cats are intermediate hosts of T. g. and less frequently kept as pets in the US) [3].

As an alternative to antibody testing, recent studies have recommended the use of a T-cell based interferon gamma release assay [17].

The most common clinical manifestations are cerebral abscesses; the lesions are frequently located in subcortical nuclear areas and multiloculated. Typical signs and symptoms include headaches at fixed locations and focal neurological deficits (hemiparesis, hemianopia, hemiataxia).

Rupture of the abscess membrane may result in diffuse meningoencephalitis, a life-threatening condition.

Magnetic resonance imaging shows ring-enhancing lesions ( $\triangleright$ Fig. 1), typically with signs of hemorrhage within the lesions which are mainly located subcortically in the basal ganglia and in the thalamus. In cerebral toxoplasmosis of the abscess-associated type, routine parameters (cells, protein, glucose, and lactate) in CSF analysis are unremarkable; polymerase chain reaction is an unreliable method in these patients. If the diagnosis cannot be established based on clinical findings and magnetic resonance imaging, the two remaining options are to undertake a treatment attempt over a period of 2 to 3 weeks or to perform a brain biopsy [3].

In diffuse meningoencephalitis, CSF analysis reveals lympho-monocytic pleocytosis of up to approximately 500 cells and slightly elevated total protein levels.

Patients with cats as pets should be advised to have their cats vaccinated against Toxoplasma gondii as a preventive measure.

Toxoplasmosis is treated with pyrimethamine plus sulfadiazine, or alternatively pyrimethamine plus clindamycin or atovaquone. Folic acid supplementation is required in patients receiving pyrimethamine.

Acute treatment (e.g. daily doses of 75-100 mg pyrimethamine and $2-4 \mathrm{~g}$ sulfadiazine) should be provided over a period of 6 to 9
- Table 3 Treatment of cerebral toxoplasmosis.

\section{Treatment}

\begin{tabular}{|l|l|}
\hline $\begin{array}{l}\text { Acute treatment } \\
\text { (6-9 weeks): }\end{array}$ & - Pyrimethamine $75-150 \mathrm{mg} / \mathrm{d}$ p.o. \\
\hline Alternative: & - Sulfadiazine $2-4 \mathrm{~g} / \mathrm{d}$ p.o. \\
& - Pyrimethamine $75-150 \mathrm{mg} / \mathrm{d}$ p.o. \\
& - Clindamycin $1800-2400 \mathrm{mg} / \mathrm{d}$ \\
& - i.v. or atovaquone .o. \\
\hline Alternative: & - Pyrimethamine $75-150 \mathrm{mg} / \mathrm{d}$ p.o. \\
\hline Adjuvant: & - Clarithromycin $2 \mathrm{~g} / \mathrm{d}$ p.o. \\
\hline
\end{tabular}

weeks, followed by maintenance therapy with pyrimethamine alone until MRI findings show substantial improvement. However, it should be noted that contrast medium enhancement in MRI images may persist for years after successful treatment of cerebral toxoplasmosis and thus is not considered the outcome parameter for treatment success or failure; resolution of focal lesions in imaging studies together with normalization of clinical findings is sufficient.

Cerebral toxoplasmosis may also be complicated by immune reconstitution inflammatory syndrome (IRIS). IRIS is characterized by worsening of symptoms despite initial response to treatment and strong contrast enhancement of the abscesses in MRI which in some cases may later spread over a wider area. The recommended treatment for IRIS is high-dose steroids.

- Table 3 provides a summary of the most common treatment options.

\section{Fungal infections}

\section{Candida albicans/Aspergillus fumigatus}

For unknown reasons, both Candida albicans and Aspergillus fumigatus infections are more frequently observed in iatrogenically immunocompromised patients compared to patients with HIV infection.

Typical signs and symptoms include fever, focal neurological deficits and preceding involvement of other organs (mucous membranes with Candida, pulmonary infection with Aspergillus) [18].

Infections with Aspergillus fumigatus frequently involve the paranasal sinuses [19]; therefore, as a rule, an otorhinolaryngologist should be consulted in all patients with Aspergillus fumigatus infection [20].

Cases of ventriculitis are rare [21].

Cranial magnetic resonance imaging reveals macroabscesses with aspergillosis and microabscesses with Candida albicans infection.

- Fig. 3 shows MRI findings typical for aspergillosis.

In CSF analysis, routine laboratory parameters are normal; however, antigen detection by means of latex antigen testing and cultures is possible.

Abscesses caused by both Candida albicans and Aspergillus fumigatus are treated with the antifungal agents amphotericin $B$, itraconazole, voriconazole or posaconazole and caspo-, mica- or anidulafungin alone or in combination; fluconazole, which is often used to treat Candida albicans, is not used to treat abscesses caused 


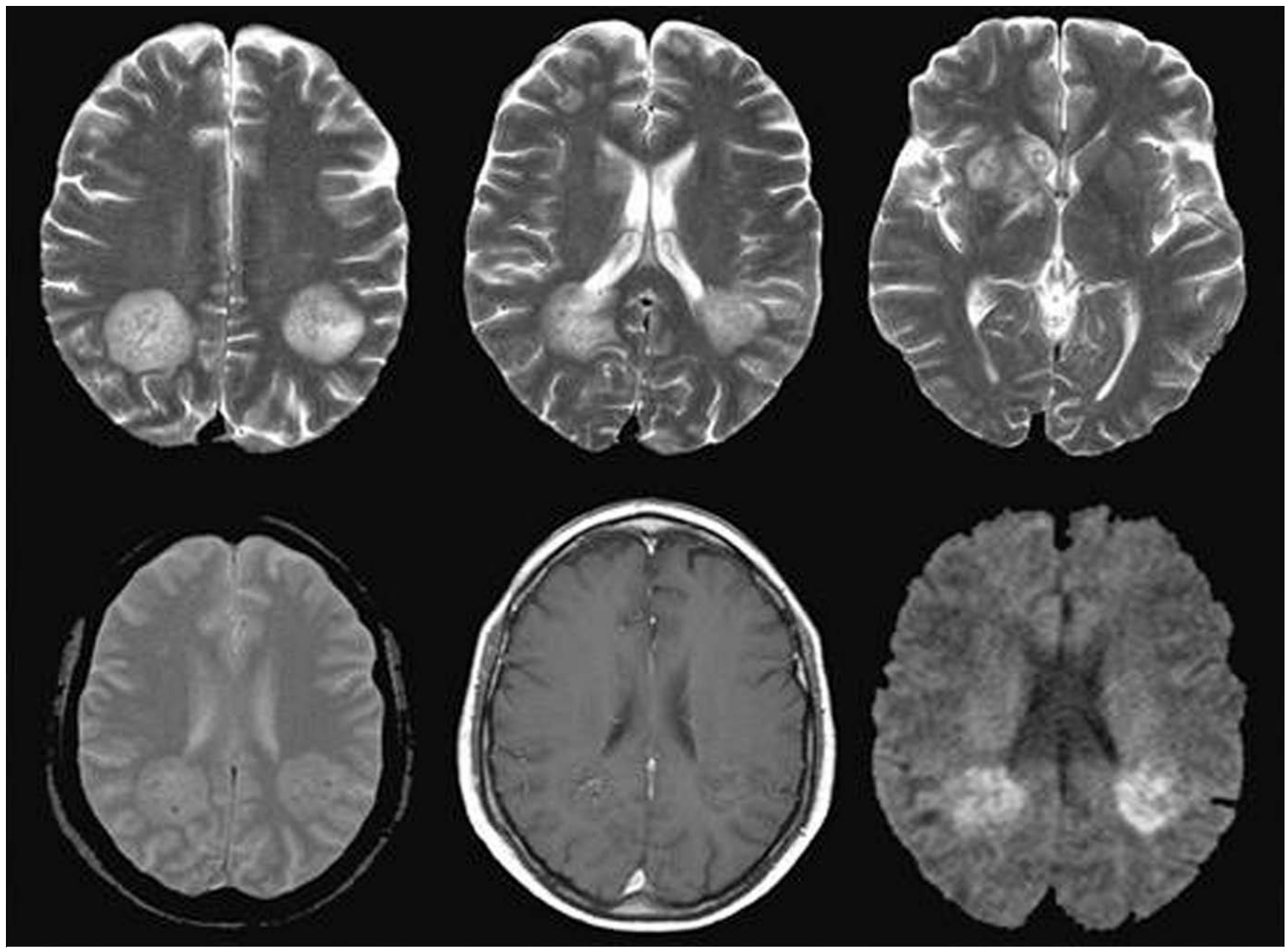

- Fig. 3 CMRI of a patient with cerebral aspergillosis (FLAIR-weighted - top - and T1-weighted with contrast medium as well as diffusion-weighted MRI; - bottom the pathogens are visible as streaks in the «tennis ball »-like space-occupying lesions.

by Aspergillus fumigatus [22]. Posaconazole can also be combined with tetrandrine, a calcium channel blocker with anti-inflammatory properties [23].

All antifungal agents have numerous side effects $[24,25]$.

\section{Cryptococcus neoformans spp}

Cryptococcus neoformans spp is the most treacherous of the fungal diseases of the immunocompromised host [26]. It takes a subacute course with diffuse headache, subfebrile temperatures and then a rapid decline in the level of consciousness, leaving the clinician little time to diagnose the underlying disease. If the diagnosis is not established within hours after the onset of consciousness level decline, the patient usually does not survive.

Typical symptoms include nuchal rigidity, bilateral papilledema and mental alteration [27].

Cerebrovascular complications are rare [28]; prognosis is determined by the increase in intracranial pressure and the development of papilledema [29-31].

Imaging shows diffuse cerebral edema, rarely microabscesses; CSF pressure is considerably increased (lumbar puncture only after CSF pressure is lowered by systemically administering e. g. manni- tol) with bilateral papilledema and CSF analysis shows lympho-monocytic pleocytosis; cryptococcosis is associated with significant complement activation, especially in HIV-negative patients [32], but not in all cases; protein and glucose levels are normal; the pathogen is detected using India ink stain (pale lavender with Gram stain) or the latex antigen test. Electron microscopy shows the typical bright mucous capsule.

Patients are treated with a combination of amphotericin B (alternatively: AmBisome), flucytosine and fluconazole until CSF tests are negative for Cryptococcal antigen; then follows maintenance treatment with fluconazole; special dosage forms of the antifungal agents help to improve the prognosis [33].

In patients with cryptococcosis, IRIS phenomena may also occur [34].

\section{Viral infections}

Herpes simplex (HSV-1 and HSV-2 meningoencephalitis) Herpes simplex encephalitis is the most common sporadic viral infection in Europe (0.2 - 0.4 cases per 100000 population) [35]. HSV- 1 is responsible for $95 \%$ of cases of fatal sporadic meningoen- 


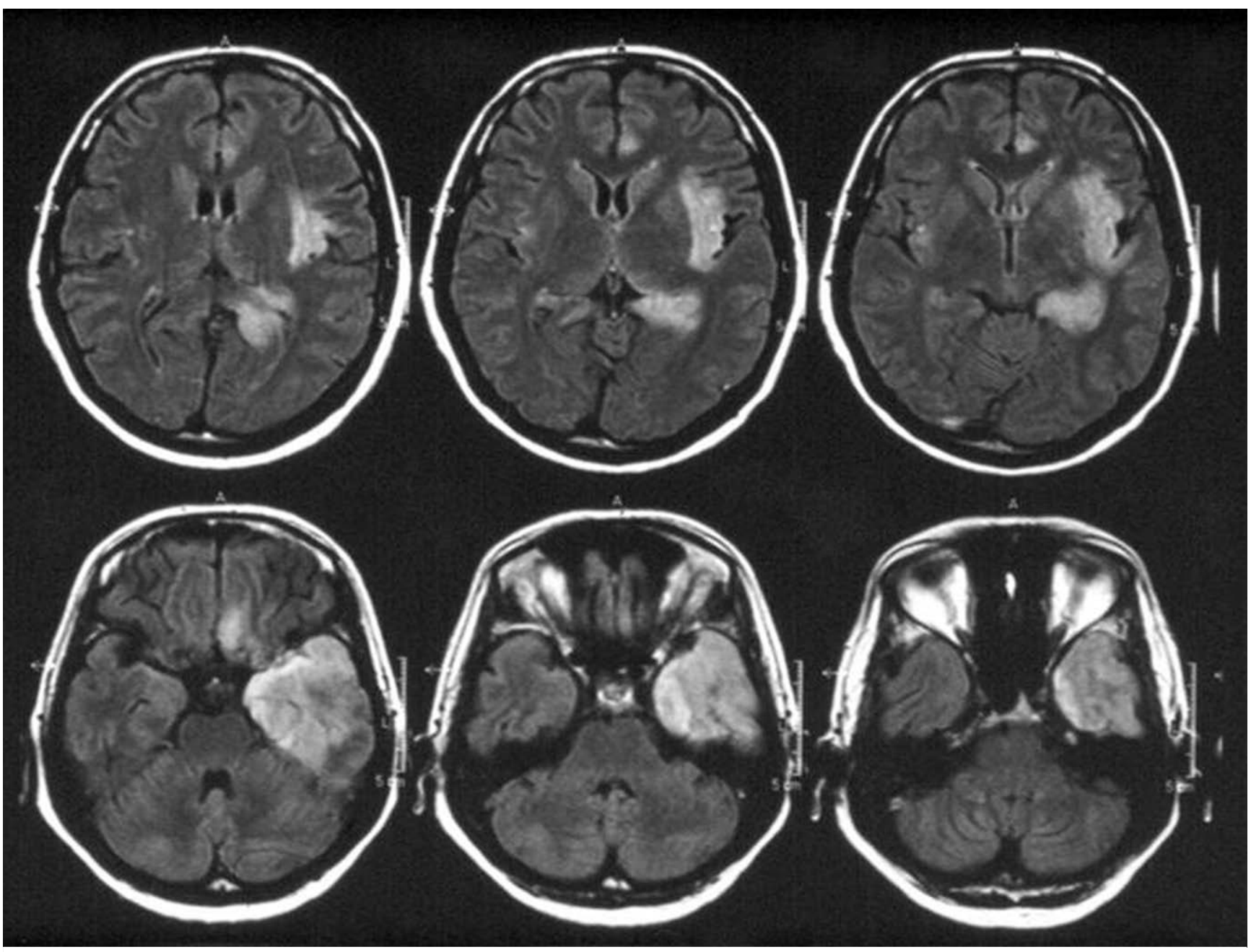

- Fig. 4 CMRI of a patients with herpes simplex virus meningoencephalitis; T2-weighted sequences reveal a large left temporal focus.

cephalitis [36]. The disease is usually caused by reactivation of latent herpes simplex virus in the body.

Signs and symptoms are similar to those observed with any other meningoencephalitis (headache, fever, meningismus, and decline in the level of consciousness).

Patients develop temporobasal or frontobasal focal encephalitis with hemorrhagic necrotic lesions and significant cerebral edema [37]. The hemorrhagic necroses can be visualized in MRI images ( $\triangleright$ Fig. 4 ).

CSF analysis reveals lympho-monocytic pleocytosis, slightly increased protein levels and HSV-positive polymerase chain reaction [38-40].

Patients with confirmed or clinically suspected HSV meningoencephalitis should be treated with acyclovir or valacyclovir $(10 \mathrm{mg} / \mathrm{kg}$ body weight/day every $8 \mathrm{~h}$ for 14 days) [41]. In contrast to immunocompetent patients, $3.8-15.7 \%$ drug-resistant virus variants are found in immunocompromised patients [42]. In case of temporal seizures, a not uncommon complication, anticonvulsant therapy should be initiated. Whether adjuvant steroid therapy improves prognosis is discussed controversially. However, since it has not been proven so far that steroid administration improves prognosis, one should refrain from adjuvant steroid use.
The introduction of antiviral therapies led to significant reduction in mortality ( $<20 \%$ ), but patients frequently experience residual neurological damage (epilepsy, mental alteration).

Especially in women, infection with HSV-2 do occasionally occur; virus reservoir is the genital tract and swabs should be taken for confirmation.

In addition, affected women often have polyradiculitis, sometimes requiring antiviral therapy for many years; famciclovir is used to prevent herpes simplex encephalitis.

\section{Cytomegalovirus infection}

Of all viruses infecting the brain, cytomegalovirus (CMV) encephalitis appears to be the one with the worst prognosis.

Initial signs and symptoms of CMV meningoencephalitis are not different from those observed with other viruses and include headache, high fever, meningismus, and rapid decline in the level of consciousness. However, over the course of the disease more than $80 \%$ of patients develop a midbrain syndrome due to infectious ventriculitis, presenting in imaging studies as marked contrast medium enhancement in ventricular ependyma; in addition, severe cerebral edema is often present [43]. Tumor-like space-occupying lesions are rarely observed. 

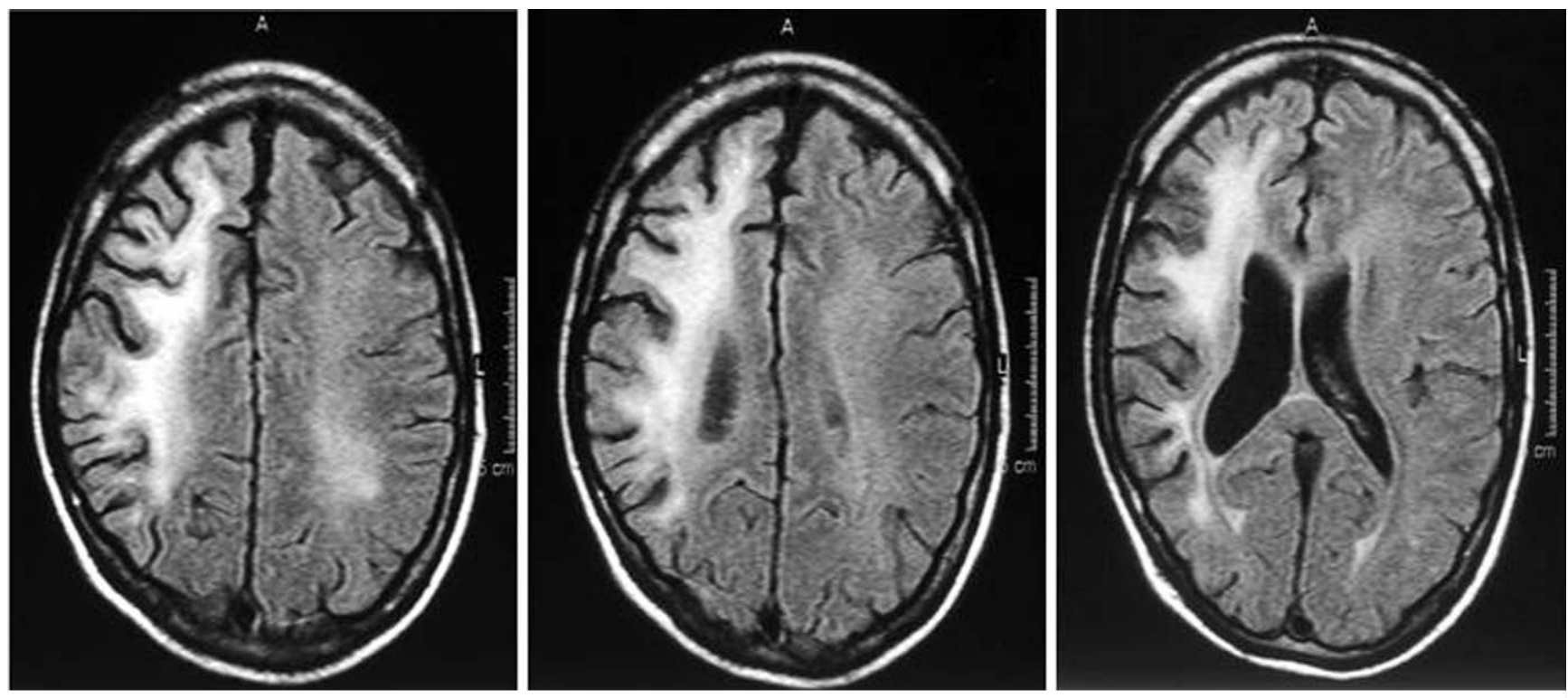

> Fig. 5 Right-hemisphere PML, starting to spread to the left side.

CSF analysis often shows granulocytic pleocytosis and significantly increased protein levels, in contrast to other viral meningoencephalitis forms with lympho-monocytic pleocytosis only; polymerase chain reaction is used for pathogen detection.

Prognosis with regard to survival is very poor (letality $>80 \%$ ). Despite the use of ganciclovir and foscarnet, surviving patients suffer from severe defects (vegetative state).

\section{Infections with JC virus}

Infections with the JC virus, a DNA polyomavirus (formerly papovavirus) targeting oligodendroglial cells and astrocytes, have grown in importance over the last few years.

JC virus infection ends in lytic demyelination of the brain.

In healthy individuals, this virus is harmless and without clinical relevance, even if it is excreted in significant numbers in urine. These harmless variants are referred to as archetype JC viruses and undergo deletions and/or re-arrangements in the regulator region of the viral genome before being detected as a pathogen in the brain [44-46]. Today, JC virus is at the center of many discussions because of its significance for immunotherapies [47, 48].

The $\mathrm{JC}$ virus-induced clinical condition is referred to as progressive multifocal leukoencephalopathy (PML). Signs and symptoms include initially mild, but then moderately to rapidly progressive focal neurological deficits and a likewise rapidly progressive mental alteration.

Cranial MRI shows a characteristic picture of multifocal demyelinating lesions with $U$-fiber involvement in subcortical white matter; within a few weeks these foci may become confluent, and affect a whole hemisphere-contrast medium enhancement is absent to minimal. A recent study proposed to use special diagnostic techniques, such as magnetic resonance spectroscopy, in unclear cases [49].

CSF analysis yields normal results, but the virus can be detected using polymerase chain reaction; thus, today brain biopsy is no longer required in most cases, but still plays a role when the diagnosis is unclear. In $20 \%$ of cases, JC virus is not detected in the CSF ob- tained at first lumbar puncture. If the speed of disease progression allows it, lumbar puncture should be repeated after about 4 weeks. At that time, another $10 \%$ of JCV infections can be detected.

Immunotherapy-induced progressive multifocal leukoencephalopathy is frequently associated with immune reconstitution inflammatory syndrome (IRIS); in this case, the MRI findings differ from those of non-IRIS PML in that significant contrast medium enhancement and massive space-occupying lesions may be present (for typical MRI findings of PML refer to - Fig. 5).

At present, there is no effective therapy for progressive multifocal leukoencephalopathy at hand. The available evidence comprises clinical single-case studies evaluating the antidepressant mirtazapine which was identified as a JCV entry inhibitor for astrocytes, and treatment attempts with maraviroc, an antiretroviral agent used in HIV therapy; in both cases, the studies are descriptive case studies, not meeting the criteria of evidence-based medicine. Patients with immunotherapy-induced PML are also treated with plasma separation.

More recently, vaccination attempts with anti-JCV capsid protein 1 (VP1) antibodies in combination with interleukin 7 and Toll-like receptor agonists have been made [50].

In multiple sclerosis research, risk stratification tools have been established in response to the PML risk in patients receiving immunotherapies, especially natalizumab; these take into account the anti- JCV antibody titers in blood, the number of previous immunotherapies, and the duration of natalizumab treatment or other immunotherapies (rituximab, dimethyl fumarate, fingolimod) [5154]. Each of these medications is associated with a different risk for PML; of these agents, natalizumab is associated with the highest risk.

Apparently, protective factors (SF2/ASF-splicing factor/alternative splicing factor) inhibiting viral replication exist in some patients [55].

Furthermore, outcome predictors have been established; negative predictors include advanced age, high numbers of JCV copies in CSF and extensive lesions in magnetic resonance imaging [56]. 


\section{CONCLUSION FOR CLINICAL PRACTICE}

With 4-29\% [8], opportunistic infections are not rare complications in iatrogenically immunosuppressed / immunomodulated patients. These are life-threatening conditions, especially at an advanced stage. It is mandatory to establish the diagnosis as early as possible. The most common pathogens in this context are rare bacteria, parasites, fungi or viruses.

\section{Conflict of Interest}

Gabriele Arendt has received research funding from Jansen-Cilag $\mathrm{GmbH}$ and ViiV Healthcare as well as lecture and/or consulting fees from Abbvie, BMS, Gilead Sciences, Jansen-Cilag and MSD. Matthias Maschke received lecture and consulting fees Komma weg from Merck. Biogen, Novartis, Roche, UCB Pharma, and Teva Pharma.

\section{References}

[1] Boucher A, Herrmann JL, Morand P et al. Epidemiology of infectious encephalitis causes in 2016. Med Mal Infect 2017; 47: 221-235

[2] John CC, Carabin H, Montano SM et al. Global research priorities for infections that affect the nervous system. Nature 2015; 527: 178-186

[3] Sundaram C, Shankar SK, Thong WK et al. Pathology and diagnosis of central nervous system infections. Patholog Res Int 2011, doi: $10.4061 / 2011 / 878263$

[4] Sonneville R, Magalhaes E, Meyfroidt G. Central nervous system infections in immunocompromised patients. Curr Opin Crit Care 2017; 23: $128-133$

[5] Shih RY, Koeller KK. Bacterial, fungal, and parasitic infections of the central nervous system: Radiologic-pathologic correlation and historical perspectives. Radiographics 2015; 35: 1141-1169

[6] Dibble EH, Boxerman JL, Baird GL et al. Toxoplasmosis versus lymphoma: cerebral lesion characterization using DSC-MRI revisited. Clin Neurol Neurosurg 2017; 152: 84-89

[7] Cunha BA. Central nervous system infections in the compromised host: a diagnostic approach. Infect Dis Clin North Am 2001; 15: 567-590

[8] Singh N, Husain S. Infections of the central nervous system in transplant recipients. Transplant Infect Dis 2000; 2: 101-111

[9] McTaggart LR, Doucet J, Witkowska M et al. Antimicrobial susceptibility among clinical Nocardia species identified by multilocus sequence analysis. Antimicrob Agents Chemother 2015; 59: 269-275

[10] Rolhion N, Cossart P. How the study of Listeria monocytogenes has led to new concepts in biology. Future Microbiol 2017; 12: 621-638

[11] Pupo I, Lepe JA, Smani Y et al. Comparison of the in vitro activity of ampicillin and moxifloxacin against Listeria monocytogenes at achievable concentrations in the central nervous system. J Med Microbiol 2017; 66: 713-720

[12] Lempp JM, Zajdowicz M], Hankinson AL et al. Assessment of the QuantiFERON-TB Gold In-tube test for the detection of Mycobacterium tuberculosis infection in United States Navy recruits. PLoS One 2017, doi:10.1371/journal.pone. 0177752

[13] Chaudhary V, Bano S, Garga UC. Central nervous system tuberculosis: an imaging perspective. Can Assoc Radiol J 2017; 68: 161-170

[14] Leonard JM. Central nervous system tuberculosis. Microbiol Spectr 2017, doi:10.1128/microbiolspec.TNMI7-0044-2017
[15] Hillemann D, Rusch-Gerdes S, Richter E. Feasibility of the GenoType MTBDRsl assay for fluoroquinolone, amikacin-capreomycin, and ethambutol resistance testing of mycobacterum tuberculosis strains and clinical specimens. J Clin Microbiol 2009; 47: 1767-1772

[16] Mai NT, Thwaites GE. Recent advances in the diagnosis and management of tuberculous meningitis. Curr Opin Infect Dis 2017; 30: $123-128$

[17] Mahmoudi S, Mamishi S, Suo X et al. Early detection of Toxoplasma gondii infection by using a interferon gamma release assay: A review. Exp Parasitol 2017; 172: 39-43

[18] Mc Carthy MW, Walsh TJ. Molecular diagnosis of invasive mycoses of the central nervous system. Expert Rev Mol Diagn 2017; 17: 129-139

[19] Starkey J, Moritani T, Kirby P. MRI of CNS fungal infections: Review of aspergillos to histoplasmosis and everything in between. Clin Neuroradiol 2014; 24: 217-230

[20] Baeesa SS, Bokhari RF, Alghamdi KB et al. Invasive aspergillus sinusitis with orbitocranial extension. Asian J Neurosurg 2017; 12: 172-179

[21] Chen TK, Groncy PK, Javahery R et al. Successful treatment of Aspergillus ventriculitis through voriconazole adaptive pharmacotherapy, immunomodulation, and therapeutic monitoring of cerebrospinal fluid (1 $\rightarrow 3$ )-B-D-glucan. Med Mycol 2017; 55: 109-117

[22] Andes D. Optimizing antifungal choice and administration. Curr Med Res \& Opinion 2013; 1-6

[23] Li SX, Song Y], Jiang L et al. Synergistic effects of tetrandrine with posaconazole against aspergillus fumigatus. Microb Drug Resist 2017, doi:10.1089/mdr.2016.0217

[24] Moreno-Rodriguez AC, Torrado-Durán S, Molero G et al. Efficacy and toxicity evaluation of new amphotericin B micelle systems for brain fungal infections. Int J Pharm 2015; 494: 17-22

[25] Tugume L, Morawski BM, Abassi M et al. Prognostic implications of baseline anaemia and changes in haemoglobin concentrations with amphotericin B therapy for cryptococcal meningitis. HIV Med 2017; 18: $13-20$

[26] George IA, Santos CAQ, Olsen MA et al. Epidemiology of cryptococcosis and cryptococcal meningits in a large retrospective cohort of patients after solid organ transplantation. Open Forum Infect Dis 2017; 4: epub ahead of print

[27] Lofgren S, Hullsiek KH, Morawski BM et al. Differences in immunologic factors among patients presenting with altered mental status during cryptococcal meningitis. J Infect Dis 2017; 215: 693-697

[28] Mishra AK, Arvind VH, Muliyil D et al. Cerebrovascular injury in cryptococcal meningitis. Int J Stroke 2017, doi: $10.1177 / 1747493017706240$

[29] Bicanic T, Brouwer AE, Meintjes G et al. Relationship of cerebrospinal fluid pressure, fungal burden and outcome in patients with cryptococcal meningitis undergoing serial lumbar punctures. AIDS 2009; 23: 701-706

[30] Qu J, Zhou T, Zhong C et al. Comparison of clinical features and prognostic factors in HIV-negative adults with cryptococcal meningitis and tuberculous meningitis: A retrospective study. BMC Infect Dis 2017, doi:10.1186/s12879-016-2126-6

[31] Rigi M, Khan K, Smith SV et al. Evaluation and management of the swollen optic disk in cryptococcal meningitis. Surv Ophthalmol 2017; 62: $150-160$

[32] Shen L, Zheng J, Wang $Y$ et al. Increased activity of the complement system in cerebrospinal fluid of the patients with Non-HIV Cryptococcal meningitis. BMC Infect Dis 2017, doi:10.1186/s12879-016-2107-

[33] Ćurić A, Möschwitzer JP, Fricker G. Development and characterization of novel highly-loaded itraconazole poly(butyl cyanoacrylate) polymeric nanoparticles. Eur J Pharm Biopharm 2017; 114: 175-185 
[34] Werner AC, Vuong LN, Hedges TR et al. Immune reconstitution inflammatory syndrome causing progressive optic nerve edema in cryptococcal meningitis. Retin Cases Brief Rep 2017, doi:10.1097/ ICB.0000000000000582

[35] Schmutzhard E. Viral infections of the CNS with special emphasis on herpes simplex infections. J Neurol 2001; 248: 469-477

[36] Kennedy PGE, Chaudhuri A. Herpes simplex encephalitis. J Neurol Neurosurg Psychiatry 2002; 73: 237-238

[37] Meyding-Lamade UK, Lamade WR, Wildemann BT et al. Herpes simplex virus encephalitis: Chronic progressive cerebral magnetic resonance imaging abnormalities in patients despite good clinical recovery. Clin Infect Dis 1999; 128: 148-149

[38] Akkaya O, Guvenc HI, Yuksekkaya S et al. Real-time PCR detection of the most common bacteria and viruses causing meningitis. Clin Lab 2017; 63: 827-832

[39] Mc Gill F, Griffiths M], Solomon T. Viral meningitis: current issues in diagnosis and treatment. Curr Opin Infect Dis 2017; 30: 248-256

[40] Van TT, Mongkolrattanothai K, Arevalo M et al. Impact of a rapid herpes simplex virus PCR assay on duration of acyclovir therapy. J Clin Microbiol 2017; 55: 1557-1565

[41] McLaughlin MM, Sutton SH, Jenson AO et al. Use of high-dose oral valacyclovir during an intravenous acyclovir shortage: A retrospective analysis of tolerability and drug shortage management. Infect Dis Ther 2017; 6: 259-264

[42] Frobert E, Burrel S, Ducastelle-Lepretre S et al. Resistance of herpes simplex viruses to acyclovir: An update from a ten-year survey in France Antiviral Res. 2014; 111: 36-41

[43] Renard T, Daumas-Duport B, Auffray-Calvier E et al. Cytomegalovirus encephalitis: Undescribed diffusion-weighted imaging characteristics. Original aspects of cases extracted from a retrospective study, and from literature review. J Neuroradiol 2016; 43: 371-377

[44] Berger JR, Miller CS, Danaher RJ et al. Distribution and quantity of sites of John Cunningham virus persistance in immunologically healthy patients: Correlation with John Cunningham virus antibody and urine John Cunningham Virus DNA. JAMA Neurol 2017; 74 : 437-444

[45] Cambron M, Hadhoum N, Duhin E et al. JCV serology in time: 3 years of follow up. Acta Neurol Scand 2017; 136: 54-58
[46] Elia F, Villani S, Ambrogi F et al. JC virus infection is acquired very early in life: Evidence from a longitudinal serological study. J Neurovirol 2017; 23: 99-105

[47] Clerico M, Artusi CA, Liberto AD et al. Natalizumab in multiple sclerosis: Long-term management. Int J Mol Sci 2017, doi:10.3390/ ijms18050940

[48] Gieselbach RJ, Muller-Hansma AH, Wijburg MT et al. Progressive multifocal leukoencephalopathy in patients treated with fumaric acid esters: A review of 19 cases. J Neurol 2017; 264: 1155-1164

[49] Schneider R, Bellenberg B, Hoepner R et al. Metabolic profiles by $1 \mathrm{H}$-magnetic resonance spectroscopy in natalizumab-associated post-PML lesions of multiple sclerosis patients who survived progressive multifocal leukoencephalopathy (PML). PloS One 2017; 12: epub ahead of print

[50] Sospedra M, Schippling S, Yousef S et al. Treating progressive multifocal leucoencephalopathy with interleukin 7 and vaccination with JC virus capsid protein VP1. Clin Infect Dis 2014; 59: 1588-1592

[51] Clavel G, Moulignier A, Semerano L. Progressive multifocal leukoencephalopathy and rheumatoid arthritis treatments. Joint Bone Spine 2017, doi: 10.1016/j.jbspin.2017.03.002

[52] Hegen H, Auer M, Bsteh G et al. Stability and predictive value of anti-JCV antibody index in multiple sclerosis: A 6-year longitudinal study. PLoS One 2017, doi:10.1371/journal.pone.0174005

[53] Plavina T, Subramanyam M, Bloomgren G et al. Anti-JC virus antibody levels in serum or plasma further define risk of natalizumab-associated progressive multifocal leukoencephalopathy. Ann Neurol 2014; 76: 802-812

[54] Schwab N, Schneider-Hohendorf T, Melzer N et al. Natalizumab-associated PML: Challenges with incidence, resulting risk, and risk stratification. Neurology 2017; 88: 1197-1205

[55] Uleri E, Ibba G, Piu C et al. JC polyomavirus expression and bell-shaped regulation of its SF2/ASF suppressor during the follow-up of multiple sclerosis patients treated with natalizumab. J Neurovirol 2017; 23: 226-238

[56] Hoepner R, Kolb EM, Dahlhaus S et al. Predictors of severity and functional outcome in natalizumab-associated progressive multifocal leukoencephalopathy. Mult Scler 2017; 23: 830-835 